\title{
Investigation of Rotor-Stator Interaction and Flow Unsteadiness in a Low Specific Speed Centrifugal Pump
}

\author{
Ning Zhang* - Minguan Yang - Bo Gao - Zhong Li - Dan Ni \\ Jiangsu University, School of Energy and Power Engineering, China
}

Instantaneous flow dynamics induced by rotor-stator interaction are detrimental to the stable operation of centrifugal pumps. In this study, unsteady rotor-stator interaction and flow structures within a low specific-speed centrifugal pump are analysed using the Large Eddy Simulation (LES) method. For that purpose, pressure pulsations and the evolution processes of vortical structures are combined to investigate rotor-stator interaction in order to clarify the inherent correlation between pressure amplitude and vorticity distribution. The results show that distinct peaks at blade passing frequency $\left(f_{B P F}\right)$ are closely associated with the positions of the monitoring point due to rotor-stator interaction. An unsteady vortical structure at the near tongue region is related to the relative position of the impeller with respect to the tongue, and the upstream effect of the volute tongue significantly affects the vorticity distribution on the blade pressure side. Rotor-stator interaction is dominated by vortex shedding in the wake of the blade trailing edge and their impingement on the volute tongue with subsequent cutting and distortion. Moreover, the high-pressure amplitude is generated with the corresponding high vorticity magnitude observed as well. Therefore, it is confirmed that pressure amplitude is significantly associated with the corresponding vorticity magnitude.

Keywords: centrifugal pump, large eddy simulation, flow unsteadiness, rotor-stator interaction, pressure pulsation, vortical structure

Highlights

- $\quad$ Numerical investigation of rotor-stator interaction and flow unsteadiness in a centrifugal pump.

- Vortical structure within the pump and its shedding from the blade trailing edge.

- Evolutionary process of vortical structure at the near tongue region.

- Correlation between pressure amplitude and vortical structure.

\section{INTRODUCTION}

Unsteady pressure pulsation due to fluid-structure interaction significantly affects the stable and safe operation of the centrifugal pump. Due to the intense rotor-stator interaction between the impeller and the volute, severe vibration can be generated causing some unexpected damage to the mechanical components, for instance, the bearing and seal of the pump [1]. Some studies have been carried out to investigate unsteady rotor-stator interaction, either by the numerical or experimental method, but most of them only focus on the unsteady pressure pulsation at blade passing frequency $\left(f_{B P F}\right)$ [2] and [3]. According to the classic theory, it is well known that flow discharged from the impeller exit, showing a jet-wake pattern, has a significant impact on rotor-stator interaction in centrifugal pumps, as well as to the unsteady pressure pulsation characteristics [4] and [5]. Therefore, it is essential to analyse unsteady flow structure, especially vorticity distribution within the pump, to clarify the influence of wake dynamics shedding from the blade trailing edge on pressure pulsations.

Posa et al. [6] and [7] numerically investigated unsteady flow distribution in a mixed flow pump using LES, especially the instantaneous vorticity distribution, and numerical results were validated with experiments. As for rotor-stator interaction, many studies only concentrate on distinct peaks in pressure spectra, especially pressure amplitude at $f_{B P F}$. Parrondo et al. [8] analysed pressure pulsations in a centrifugal pump for various conditions, and particular emphasis was placed on pressure amplitude at $f_{B P F}$. With the development of non-contact and nonintrusive measuring techniques in recent years, unsteady particle image velocimetry (PIV) and laser Doppler velocimetry (LDV) measuring techniques are often applied to investigate complex rotor-stator interaction in pumps so that no external disturbance would be imposed on the flow field. Keller et al. [9] used PIV to analyse unsteady flow field around the near tongue region at high flow rates, and consecutive contours of vorticity sheet shedding in the wake of the blade trailing edge were attained to observe the details of flow evolution at the tongue region. $\mathrm{Wu}$ et al. [10] and [11] also applied the PIV technique to investigate the internal flow field in a centrifugal pump. Stress was laid on the flow distribution at a nominal flow rate, and an unsteady velocity field together with principal Reynolds normal stress and the principal Reynolds shear stress were revealed. Feng et al. [12] successfully used LDV to periodically measure unsteady flow in a radial flow pump, and complex flow structures in the interaction region 
between the rotating impeller and stationary diffuser were captured. To alleviate the intense rotor-stator interaction, some effective approaches could be implemented, for instance increasing the radial gap between the impeller and volute [13], optimal design of the impeller and the volute and some specially devised structures of the pump [14]. However, in these studies, most simply concentrate on pressure amplitude at $f_{B P F}$ or unsteady flow structure individually, and a combination analysis has rarely been conducted. As a result, a comprehensive understanding of rotorstator interaction, especially the correlation between instantaneous flow dynamics and pressure pulsation, has not been thoroughly illustrated.

In this study, unsteady rotor-stator interaction in a low specific speed centrifugal pump is analysed using a numerical method. Pressure pulsation signals together with vorticity distribution are attained. Special attention is laid on the vortical structure shedding in the wake of the blade trailing edge and the interaction with the volute tongue. The detailed evolution process of vortical structures at the near tongue region and within the blade channel are focused and analysed. Finally, the correlation between pressure amplitude and vorticity distribution is discussed.

\section{NUMERICAL INVESTIGATION}

\subsection{Governing Equations}

After adding a filter to Navier-Stokes (N-S) and continuity equations, LES governing equations can be described in the following form:

$$
\begin{gathered}
\frac{\partial \bar{u}_{i}}{\partial x_{i}}=0, \\
\frac{\partial \bar{u}_{i}}{\partial t}+\frac{\partial}{\partial x_{j}}\left(\bar{u}_{i} \bar{u}_{j}\right)= \\
=-\frac{1}{\rho} \frac{\partial \bar{p}}{\partial x_{i}}+\frac{\partial}{\partial x_{j}}\left[v\left(\frac{\partial \bar{u}_{i}}{\partial x_{j}}+\frac{\partial \bar{u}_{j}}{\partial x_{i}}\right)\right]+\frac{\partial \bar{\tau}_{i j}}{\partial x_{j}}+S_{i},
\end{gathered}
$$

where $\bar{u}_{i} \quad(i=1,2,3)$ is the grid-scale velocity component, $\bar{p}$ is the grid-scale static pressure, $\rho$ is the density and $v$ is the kinematic viscosity. $S_{i}$ is the source term, and $\bar{\tau}_{i j}$ is the subgrid-scale (SGS) stress tensor having the form of $\overline{\tau_{i j}}=\overline{u_{i}} \bar{u}_{j}-\overline{u_{i} u_{j}}$. From comparisons with conventional Navier-Stokes (N-S) equations, it is found that an additional SGS stress tensor $\bar{\tau}_{i j}$ term is introduced in the LES governing equations. In the present study, SGS model Smagorinsky-Lilly is applied to close the equations and $\bar{\tau}_{i j}$ term is solved in Eq. (3).

$$
\bar{\tau}_{i j}-\frac{1}{3} \delta_{i j} \bar{\tau}_{k k}=-2 v_{T} \bar{S}_{i j},
$$

where $\bar{S}_{i j}$ is the strain rate tensor and $v_{T}$ is the SGS stress viscosity having the form of Eq. (4).

$$
\begin{gathered}
v_{T}=\left(C_{s} \Delta\right)^{2}|\bar{S}|, \\
|\bar{S}| \equiv \sqrt{2 \overline{S_{i j} \bar{S}_{i j}},}
\end{gathered}
$$

where $\Delta$ is the filter scale and $C_{S}$ is a dimensionless parameter called the Smagorinsky coefficient. Moreover, during numerical calculation, $C_{s}$ is usually a constant 0.1 .

\subsection{Mesh Generation}

A low specific speed $n_{s}=69$ centrifugal pump, incorporating a two-dimensional impeller with six backward-curved blades, is designed for investigation. Moreover, the main parameters of the model pump are presented in Table 1. During numerical simulation, the front and back chambers of the model pump are usually neglected for simplification. However, in low specific speed pumps, the leakage flow from the wear ring clearance is normally more than $5 \%$ of the total flow rate, and it would have a significant effect on the unsteady flow structure inside the model pump. Therefore, in this paper, the entire computational domain, containing inlet suction, impeller, volute, pump outlet, front and back chambers, is established for calculation as shown in Fig. 1. Moreover, the wear ring clearance is $0.5 \mathrm{~mm}$.

Table 1. Main design parameters of the model pump

\begin{tabular}{lc}
\hline Parameters & value \\
\hline Nominal flow rate $Q_{d}$ & $55 \mathrm{~m} / \mathrm{h}$ \\
\hline Designed head $H_{d}$ & $20 \mathrm{~m}$ \\
\hline Nominal rotating speed $n_{d}$ & $1450 \mathrm{r} / \mathrm{min}$ \\
\hline Specific speed $n_{s}=3.65 n_{d} \sqrt{Q_{d}} / H_{d}{ }^{0.75}$ & 69 \\
\hline Blade number $Z$ & 6 \\
\hline Angle of volute tongue & $20^{\circ}$ \\
\hline Impeller suction diameter $D_{1}$ & $80 \mathrm{~mm}$ \\
\hline Impeller outlet diameter $D_{2}$ & $260 \mathrm{~mm}$ \\
\hline Impeller outlet width $b_{2}$ & $17 \mathrm{~mm}$ \\
\hline Volute inlet diameter $D_{3}$ & $290 \mathrm{~mm}$ \\
\hline Volute outlet diameter $D_{4}$ & $80 \mathrm{~mm}$ \\
\hline Tangential velocity at impeller exit $u_{2}$ & $19.6 \mathrm{~m} / \mathrm{s}$ \\
\hline Impeller rotating period $\Delta T$ & $0.0414 \mathrm{~s}$ \\
\hline
\end{tabular}




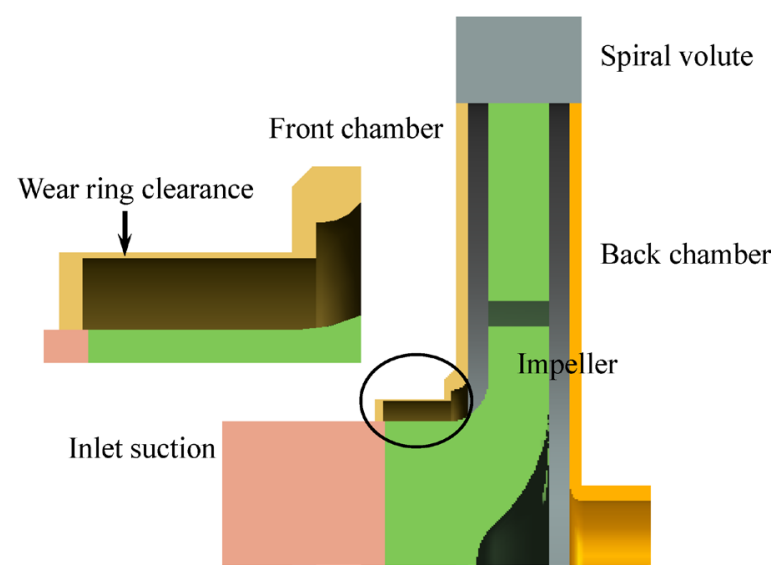

Fig. 1. Computational domain of the model pump

Structured grids of the model pump are generated using the Ansys-ICEM mesh generation tool for high precision calculation. Fig. 2 shows partially structured grids at the mid-span of the impeller. In the near-wall region, a fine grid is required by LES, so grid cells near the wall are refined to satisfy the requirement [15] to [17]. At the near wall region, the mesh cell size is lower than $0.5 \mathrm{~mm}$. Finally, the averaged $y^{+}$value of the whole computational domain is approximately 4.5 , which would provide adequate resolution in the critical regions of the computation domain. After the independent mesh check, the performance of the model pump does not change more than $0.5 \%$ when the overall mesh element exceeds $2 \times 10^{6}$. Finally, the overall mesh element used in the calculation is about $2.5 \times 10^{6}$. The mesh element of each part, i.e. inlet and outlet suctions, impeller, volute casing, front chamber, back chamber, is 58,$240 ; 56,128 ; 1,298,386 ; 752,361$; 270,$668 ; 127,372$, respectively. The same mesh is used for steady and transient simulation.

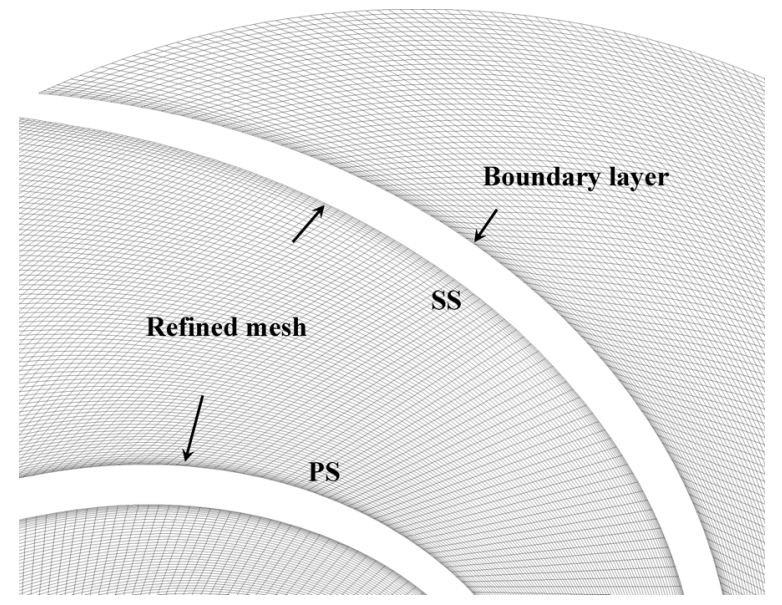

Fig. 2. Structured grids at the mid-span of the impeller

\subsection{Solution Parameters}

Commercial software Ansys-Fluent 13.0 is used for numerical simulation. For transient LES calculation, steady numerical simulation results achieved by the RNG $k-\varepsilon$ model are set as the initialization condition. Velocity inlet boundary condition is imposed at the pump inlet suction, where a spectral synthesizer is chosen for fluctuating velocity algorithm.

A constant pressure $p=1 \times 10^{5} \mathrm{~Pa}$ boundary condition is imposed at the pump outlet. To achieve adequate resolution of pressure signals, the time step is set as $\Delta t=1.15 \times 10^{-4} \mathrm{~s}$. During numerical simulation, when the continuity residual is lower than $3 \times 10^{-5}$, the result is considered to be converged. Nearly 30 impeller revolutions are calculated to enable periodic pressure pulsation results. Moreover, the inside faces of the front and back chambers rotate synchronously with the rotating impeller.

It is well accepted that even at a nominal flow rate, flow field distribution along the volute periphery tongue casing is not circumferentially uniform leading to pressure pulsating [18] and [19]. Therefore, it is essential to have a comprehensive understanding of pressure pulsation along the volute. For this purpose, twenty monitoring points are evenly mounted on the volute casing near the impeller exit, as seen in Fig. 3. The angle between two adjacent monitoring points is $18 \mathrm{deg}$. The angle of the volute tongue is $20 \mathrm{deg}$, and it is located between Point B and Point C. Pressure pulsation signals are processed with a FFT (Fast Fourier Transform) algorithm to investigate pressure spectrum characteristics under various flow rates.

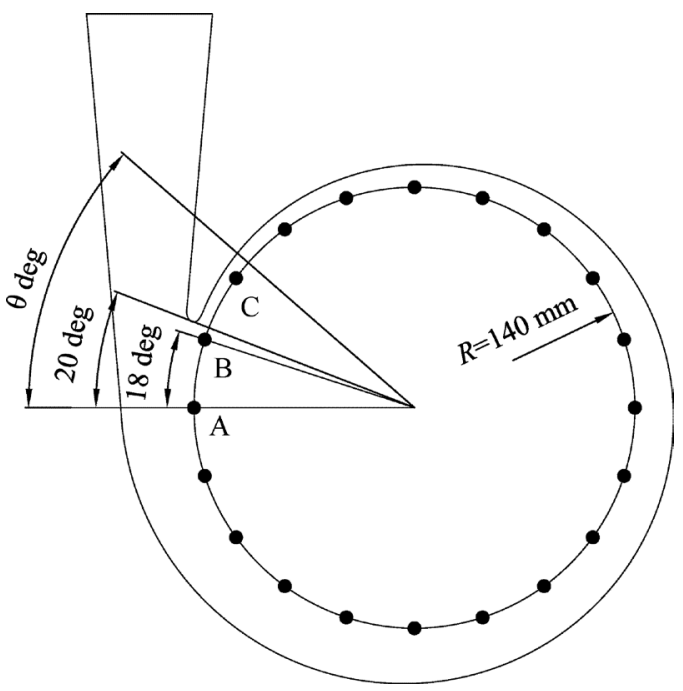

Fig. 3. Details of the monitoring point on the volute casing 


\section{EXPERIMENTAL SETUP}

To validate the accuracy of the current numerical method, experimental investigation of the model pump was conducted on a closed test rig as shown in Fig. 4. An electronic flowmeter was applied to obtain flow rates of the model pump at various operating conditions; meanwhile, the head was measured using the pressure gauges located at the pump inlet and outlet pipes. The measuring errors are lower than $1 \%$ of the measured values. During experiments, the rotating speed of the model pump was ensured to be at the design value of $1450 \mathrm{r} / \mathrm{min}$ by adopting a frequency inverter. Meanwhile, one pressure transducer (PCB113B27 series) is mounted at Point C to achieve the pressure spectrum. During experiments, the inlet static pressure is about atmospheric pressure. Even at $1.4 Q_{d}$, the critical NPSH is about $3.3 \mathrm{~m}$, which means that cavitation could not occur during pump operations.

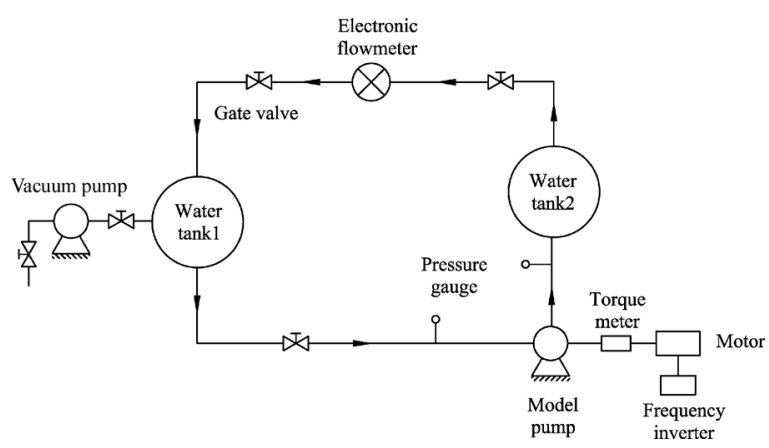

Fig. 4. Closed test platform

\section{RESULTS AND DISCUSSIONS}

\subsection{Validation of the Numerical Method}

To validate the accuracy of the current numerical method, Fig. 5a shows a performance comparison between numerical and experimental results. The best efficiency point is around $1.1 Q_{d}$. The predicted results exhibit good agreement with the experimental results. From $0.4 Q_{d}$ to1.4 $Q_{d}$, calculation errors are lower than $2 \%$. At flow rates lower than $0.4 Q_{d}$, numerical error increases, rising to $3.5 \%$ from head curve at $0.2 Q_{d}$. It remains a satisfactorily precise calculation. As observed from Fig. 5b, the calculation error at $f_{B P F}$ is relatively small: normally lower than $10 \%$. As for pulsation pressure prediction, it is a fairly good result [20] and [21]. Numerical result shows that leakage flow from the wear ring clearance is almost
$10 \%$ of the total flow rate at the design flow rate, and it increases to $19 \%$ of the total flow rate at 0.2 $Q_{d}$. Therefore, it is unreasonable to exclude the front chamber in low specific speed centrifugal pumps, since the disturbance flow at the impeller inlet leaking from the wear ring clearance would generate apparent influence on the unsteady flow field.
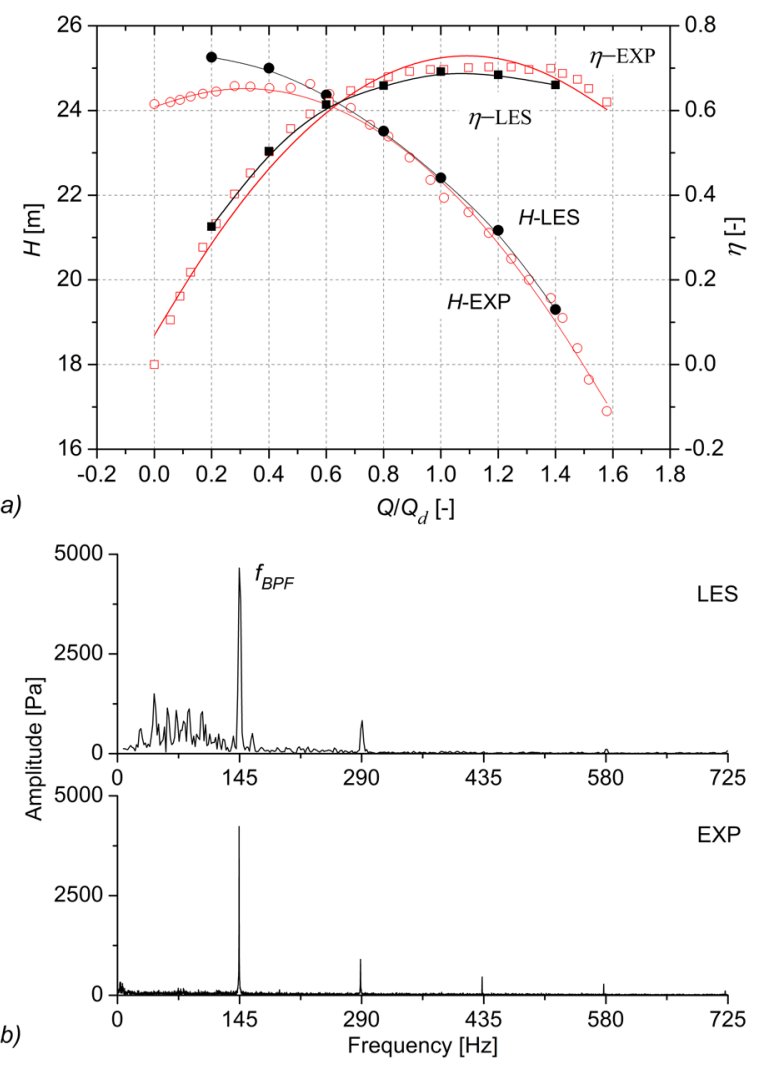

Fig. 5. a) Performance comparison between experimental and numerical results, b) comparison of pressure spectra

From the comparison results, it indicates that the current numerical method could effectively capture the main flow structures within the model pump.

\subsection{Unsteady Pressure Pulsations}

Fig. 6a shows the time domain pressure signals of Point $\mathrm{C}$ for numerical simulation, and the corresponding pressure spectra are shown in Fig. 6b. It is found that pressure signals fluctuate significantly, and pronounced peaks at blade passing frequency $f_{B P F}$ $(145 \mathrm{~Hz})$ dominate the pressure spectra under different flow rates. Meanwhile, the high harmonic frequency $2 f_{B P F}$ could also be identified.

According to Fig. 6b, discrete peaks at $f_{B P F}$ are the predominant components in pressure spectra inferred 
from the numerical results. Due to the asymmetry of the spiral volute and jet-wake flow pattern at the blade exit, flow field is not circumferentially uniform, and it would affect pressure spectra at different monitoring points along the volute casing. To clarify the correlation, Fig. 7 presents angular distributions of pressure amplitudes at $f_{B P F}$ under various flow rates. It is observed that angular distribution of pressure amplitude at $f_{B P F}$ exhibits a modulated pattern characterized by the presence of six local peaks and valleys. The modulation pattern is closely associated with rotor-stator interaction inside the model pump [22] and [23]. At off-design conditions, especially at flow rates of $0.2 Q_{d}$ and $1.4 Q_{d}$, pressure amplitudes are much larger than that at the rated condition.
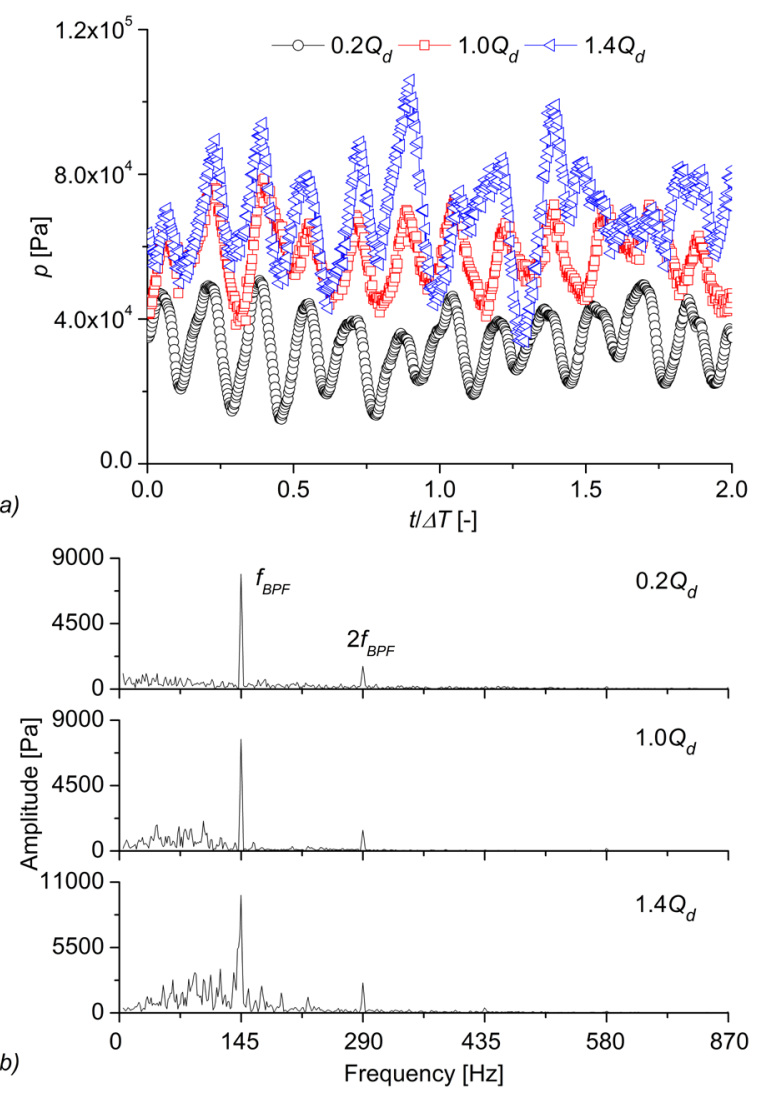

Fig. 6. a) Time domain pressure pulsation signals at point $C$ under three flow rates, $b$ ) the corresponding pressure spectra

From Fig. 7, it is also noted that pressure amplitudes at the near tongue region, Points A, B, and $\mathrm{C}$, show great discrepancy, and Fig. 8 presents the enlarged configuration of pressure amplitudes at $f_{B P F}$ for a nominal flow rate. As observed, pressure amplitude at Point $\mathrm{B}(\theta=18$ deg ahead of the volute tongue) is quite small. In contrast, pressure amplitude at the point after the volute tongue, around Point $\mathrm{C}(\theta$

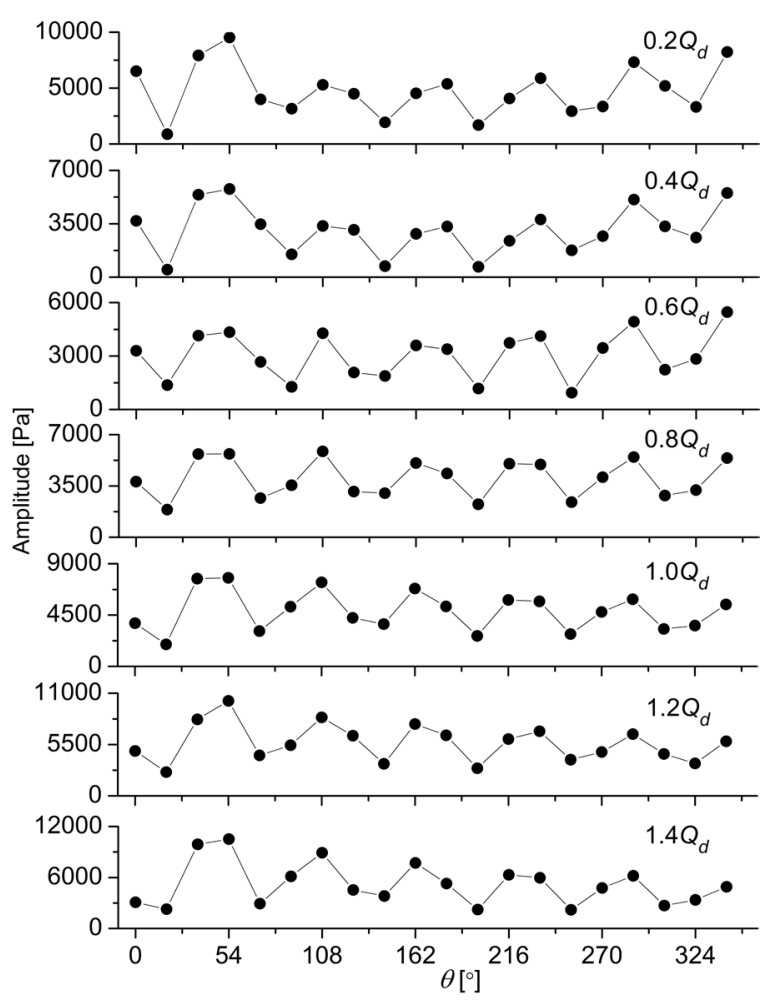

Fig. 7. Angle distributions of pressure amplitudes at fBPF along the volute casing under various flow rates

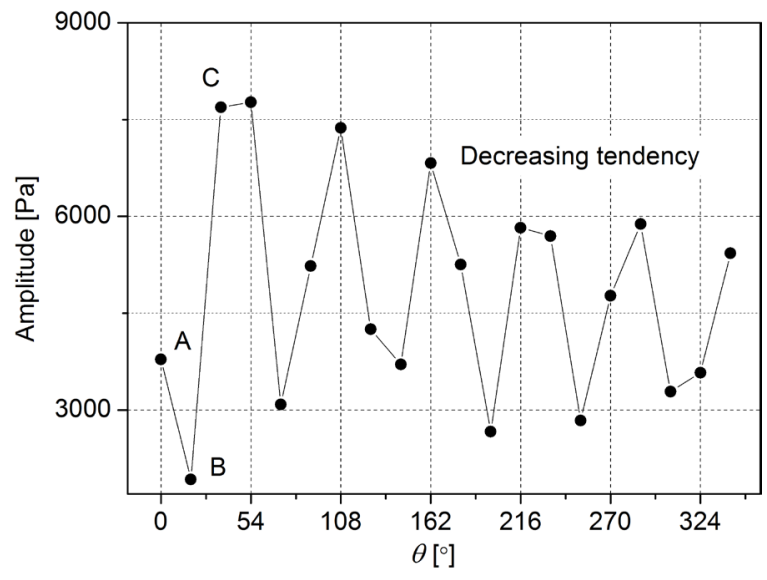

Fig. 8. Pressure amplitudes at fBPF for nominal flow rate

= $54 \mathrm{deg}$ ), achieves maximum, indicating that rotorstator interaction is more intense around this region. This phenomenon is more obvious when the model pump operates at low flow rates of $0.2 Q_{d}$ and 0.4 $Q_{d}$. At a great distance from the volute tongue $(\theta>$ $54 \mathrm{deg}$ ), rotor-stator interaction is less intense due to the increasing radial gap between the impeller and volute. As shown in Fig. 8, it is evident that pressure amplitudes $(\theta>54 \mathrm{deg})$ show a decreasing tendency 
due to the less significant rotor-stator interaction. In these regions, pressure pulsation is mainly induced by the unsteady flow structure shedding from the blade trailing edge (jet-wake pattern).

\subsection{Instantaneous Flow Dynamics}

Based on the results of Fig. 7 and Fig. 8, it is clear that pressure amplitudes on the periphery of the spiral volute casing show a significant difference. Around the volute tongue region, pressure amplitude at Point $\mathrm{C}$ is much larger than that at Point $\mathrm{B}$, as means that flow induced pulsation pressure mechanisms at these regions are obviously different. Therefore, it is essential to clarify flow structures inside the model pump and its influence on pressure amplitude, particularly around the volute tongue zone, where intense fluid-structure interaction is expected.

Fig. 9 shows relative velocity distributions on the mid-span of the impeller for four flow rates, namely $0.2 Q_{d}, 0.6 Q_{d}, 1.0 Q_{d}$ and $1.4 Q_{d}$. As observed, at a high flow rate of $1.4 Q_{d}$, flow mainly concentrates on the blade suction side due to the increasing blade inlet angle, and the high-velocity region almost covers the whole blade passage, extending to the blade exit. At almost half chord of the blade pressure side, a significant low relative velocity region is formed. Meanwhile, a small portion of the flow detaches from the blade surface in this region. At a low flow rate of $0.6 Q_{d}$, the separate flow region on the blade pressure side expands, forming a significant counter-clockwise rotating vortex. At the blade exit, the velocity of the flow near the blade pressure side is much larger than that near the blade suction side. Hence, flow at the impeller outlet is characterized by a jet-wake pattern, which results in pressure pulsating at any position along the spiral volute casing. At $0.2 Q_{d}$, due to the reverse flow at the blade exit, a large scale vortex occurs on the blade pressure side in Channel 2 causing partial blade channel blockage. In general, at low flow rate, flow separation may easily occur inside the blade channels due to the inlet flow deviating seriously from the rated condition. As observed in Channel 1, flow distribution is in a disorderly status characterized by many small scale vortices developed. At a nominal flow rate, flow distribution is more uniform than that at off-design conditions, especially at the blade outlet region. However, the separate flow region on the blade pressure side could still be observed. Due to the high incident angle, flow tends to move towards the blade suction side, and low-velocity magnitude on the blade pressure side is expected. Furthermore, the curvature of the blade may be larger, and fluid could not move along the blade streamlines. Consequently, a highpressure gradient occurs, leading to secondary flow forming. Finally, a separate flow structure develops on the blade pressure side due to the combination effects.

$$
\text { Vorticity }=2 \omega_{z}=\left(\frac{\partial v_{y}}{\partial x}-\frac{\partial v_{x}}{\partial y}\right)
$$

Having investigated relative velocity inside the blade channels at four flow rates, vorticity distribution would be further analysed to illustrate instantaneous flow dynamics. Fig. 10 shows the vorticity magnitude at the mid-span of the impeller for a nominal flow rate. As observed, four typical vorticity regions with high magnitude developed inside the model pump, specifically regions $\alpha, \beta, \gamma$, and $\delta$. At the blade pressure side of each channel, high vorticity magnitude region $\beta$ is observed, in accordance with the flow detachment region as shown in Fig. 9. The vorticity value in the central zone of the impeller is quite small indicating that the flow field is relatively uniform. On the blade suction side, another strong vorticity region $\gamma$ is generated, and it almost starts from the blade leading edge covering the whole blade chord. This high vorticity region is probably caused by the boundary layer developed on the blade suction side, and a high-velocity gradient is usually expected in this region. The vorticity sheet shows good coherence on the blade before approaching the blade trailing edge. At the blade outlet, it is evident that the vortical structure in region $\gamma$ starts to detach from the blade suction side, consequently shedding in the wake. Then the shed vortical structure moves into the volute casing; therefore, a high vorticity magnitude region $\delta$ is generated inside the volute casing. The periodic shedding effect of the vortical structure would cause intense pressure changes and pulsations, as characterized by the modulated pattern shown in Fig. 7. At the near tongue area, it is noted that a rather strong vorticity region $\alpha$ is generated. It is caused by the impingement effect between the shedding vortex from the blade trailing edge and the volute tongue. Due to the impingement effect, turbulence activity in this region is more intense, as manifested by the increase of vorticity magnitude, and it would have a significant influence on pressure pulsations in this region.

Due to the intense impeller-tongue interaction, the flow structure around the near tongue region is rather complicated and distorted. As observed in Fig. 7, pressure amplitudes at the near tongue region, Points A, B, and C, show significant discrepancy. The pressure magnitude at Point $\mathrm{C}$ is almost 4 times that at Point B. Besides, in Fig. 10, vorticity magnitudes 

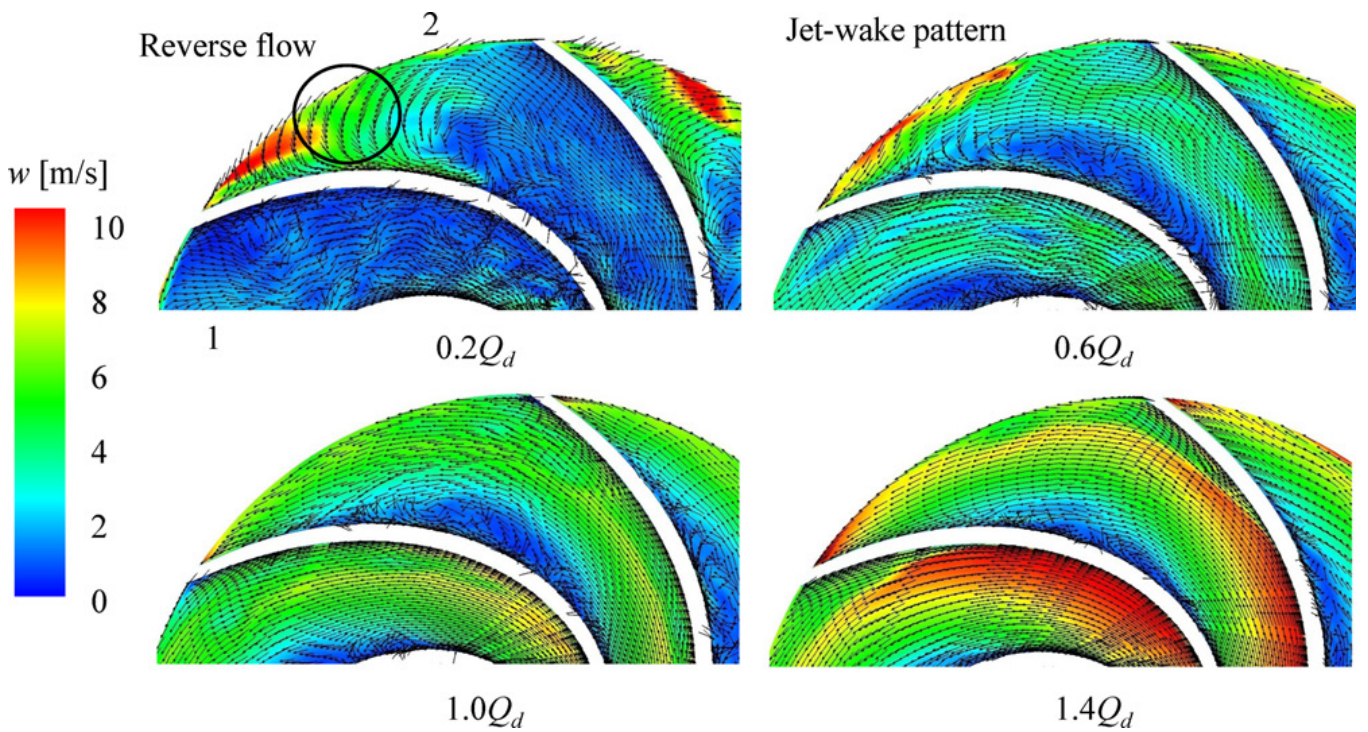

Fig. 9. Relative velocity distributions on mid-span of the impeller for different flow rates

in the areas before and after the volute tongue differ obviously, and a high vorticity magnitude area is generated at the after tongue region. Therefore, the emphasis is placed upon the vorticity distribution at the interested near tongue region to investigate instantaneous flow dynamics and clarify the correlation between vortical structure and pressure amplitude.

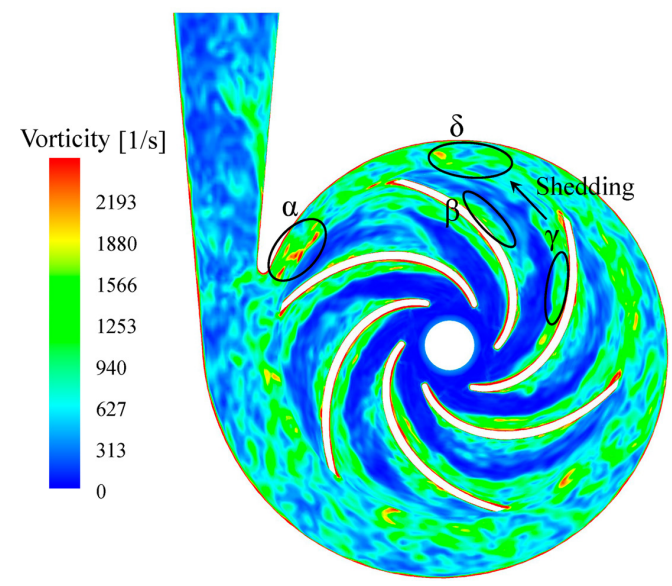

Fig. 10. Vorticity distribution at the mid-span of the impeller under nominal flow rate

Fig. 11 shows instantaneous vorticity distributions for four consecutive positions of the impeller at the rated condition. At $t_{1}=0$ (Fig. 11a), it is evident that two vortical structures, denoted as VS1 and VS2, are generated around the volute tongue. These vortical structures are closely associated with the wake shedding from the trailing edge of Blade
3. When approaching the volute tongue, the vortical structure impinges on the tongue, and it is cut into two parts (VS1 and VS2). Then the two resultant vorticity zones continue their motion towards the pump exit and the narrow side of the tongue respectively. In Fig. 11b, when Blade 2 aligns with the tongue, the torn-off portion, VS1 moves to the pump outlet and experiences a reduction in magnitude. It is more significant that seen in Fig. 11d, and VS1 becomes progressively weaker, while undergoing some distortion. This is due to the combination effect of the bending and streamwise stretching of the vortical structure near the volute tongue and its mixing effect with the external flow with lower vorticity magnitude in the diffuser section. Finally, with the impeller rotating, VS1 would dissipate in the diffuser section. It is also noted that the magnitude of VS2 undergoes a rapid decrease at this moment. This is due to the increasing of the radial distance between the impeller and the volute when VS2 continues its motion to the narrow side of the volute tongue. In Fig. $11 \mathrm{c}$, with the impeller continuously rotating, Blade 2 moves away from the volute tongue. However, the wake shedding from the trailing edge of Blade 2 has not reached to the tongue; therefore, the intense impingement effect does not occur. Consequently, the low vorticity magnitude around Point B is always observed.

According to the results of Fig. 11, vortical structures around the volute tongue that show obvious differences, which cause different pressure amplitudes at Points B, and C. Due to the impingement effect of vortical structure on the tongue, a high magnitude of 


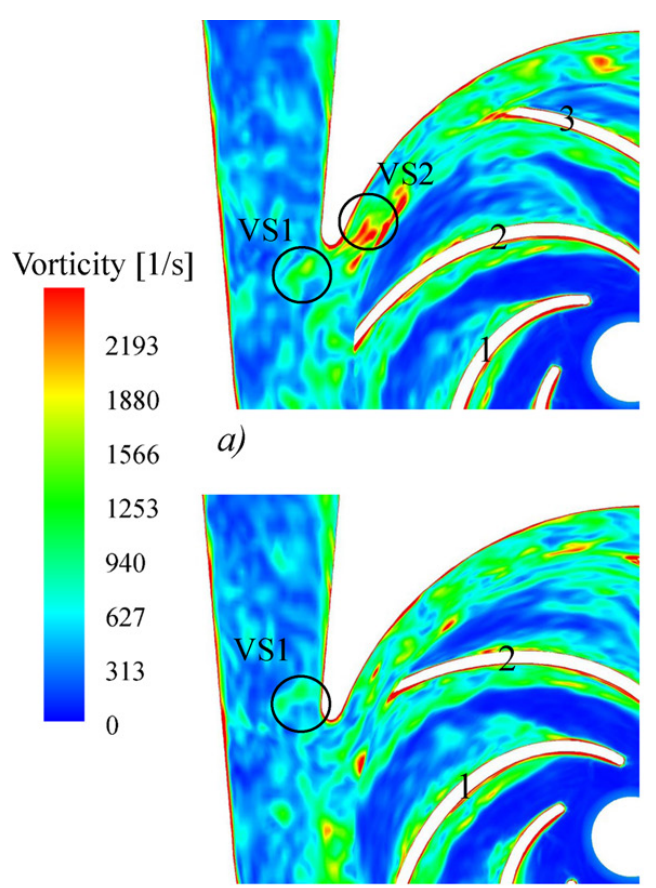

c)

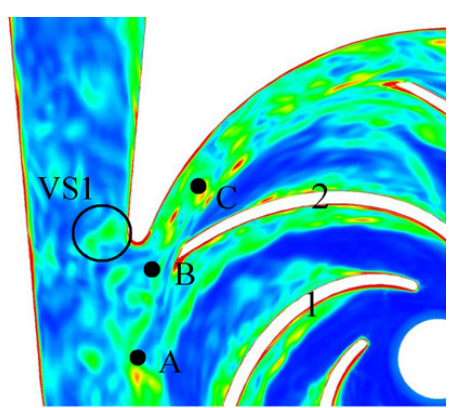

b)

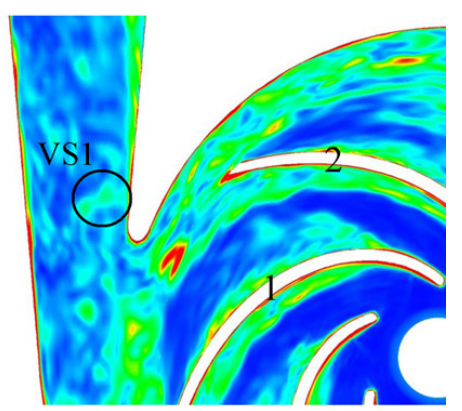

d)

Fig. 11. Configurations of vorticity distributions around the volute tongue at the mid-span of the impeller for four consecutive positions at nominal flow rate; a) $\left.\left.\left.t_{1}=0, b\right) t_{2}=18 / 360 \Delta T, c\right) t_{3}=28 / 360 \Delta T, d\right) t_{4}=38 / 360 \Delta T$

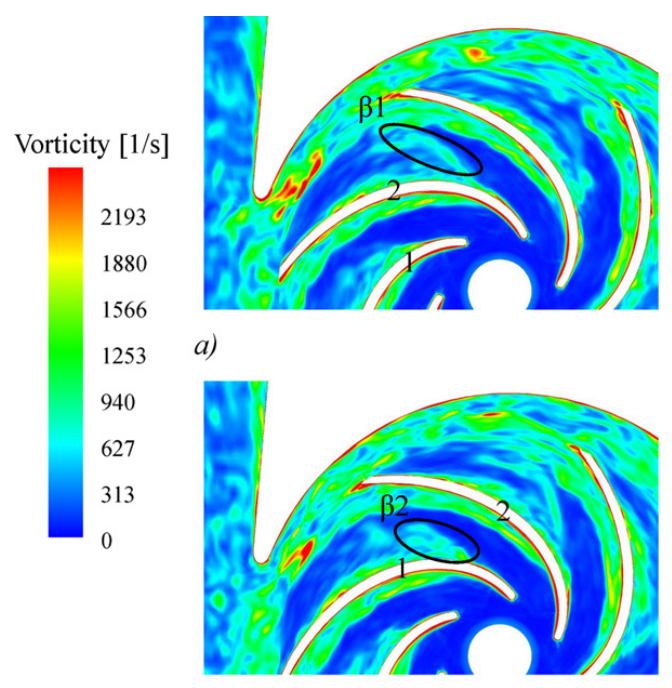

c)

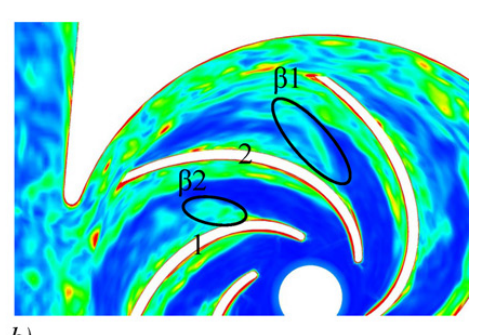

b)

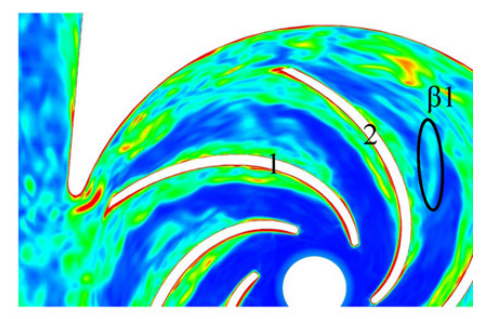

d)

Fig. 12. Detailed analysis of vorticity distributions inside the blade channels for four consecutive positions at nominal flow rate; a) $t_{1}=0$, b) $t_{2}=28 / 360 \Delta T$, c) $\left.t_{3}=48 / 360 \Delta T, d\right) t_{4}=78 / 360 \Delta T$

the vorticity region VS2 is generated. It also means that strong turbulence activity is expected in this region as indicated by the high value of vorticity magnitude, and the corresponding Point $\mathrm{C}$ coincidentally is located in this area. As a contrast, Point B would always undergo lower vorticity magnitude with the impeller rotating periodically. Therefore, it is certain that the pressure amplitudes of the monitoring point are closely associated with the corresponding vorticity magnitudes, and high vorticity magnitude would generally result in high-pressure amplitude.

The unsteady rotor-stator interaction has two kinds of effects, as discussed by Feng et al. [12]. The first is the downstream effect of the impeller acting on 


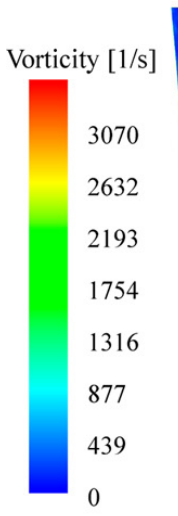

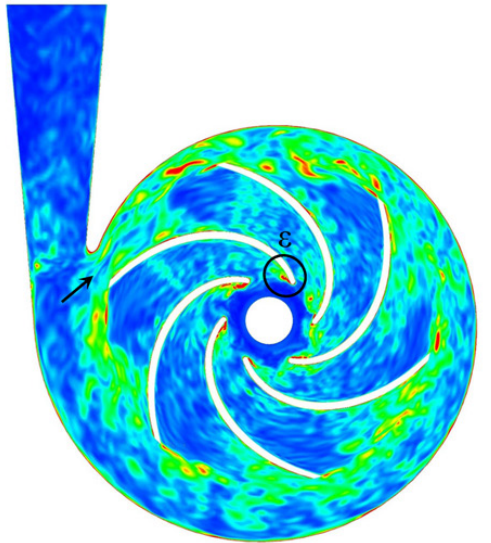

$0.2 Q_{d}$

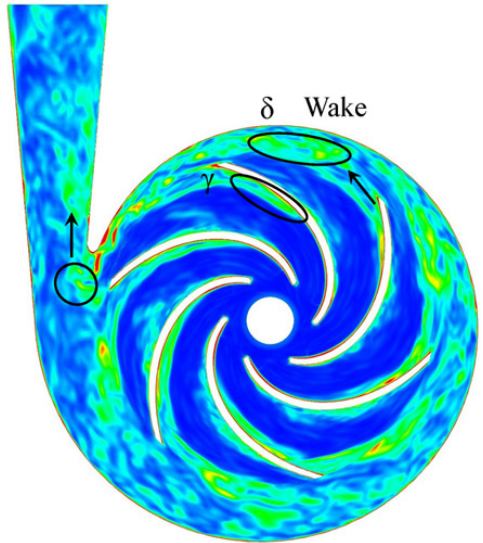

$1.4 Q_{d}$

Fig. 13. Vorticity magnitudes at off-design flow rates of $0.2 Q_{d}$ and $1.4 Q_{d}$

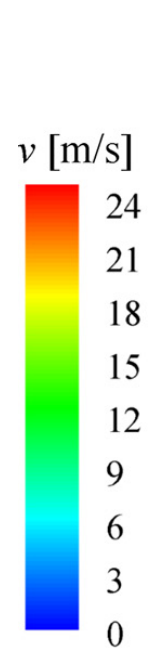

$0.2 Q_{d}$

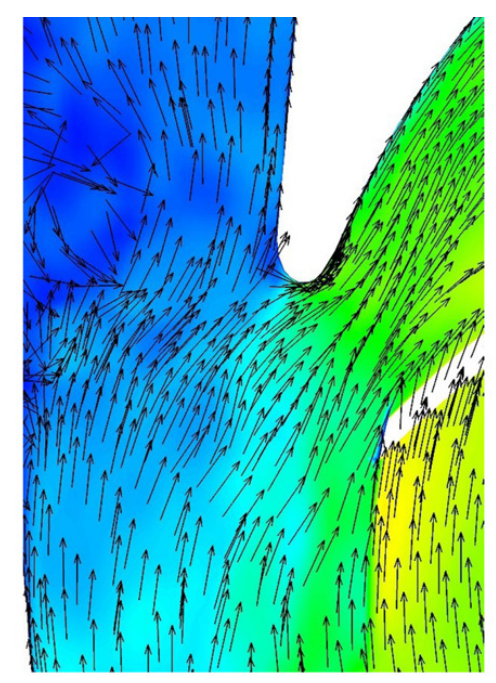

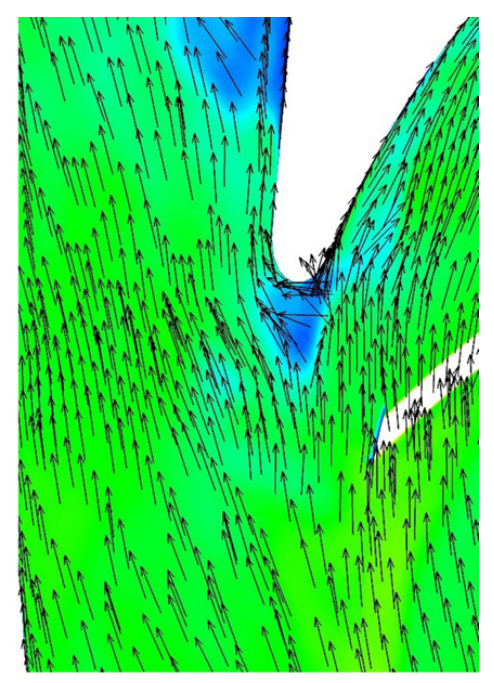

$1.4 Q_{d}$

Fig. 14. Absolute velocity distributions around the volute tongue for flow rates of $0.2 Q_{d}$ and $1.4 Q_{d}$

the stator characterized by the unsteady effects due to flow discharged from the impeller and the jet-wake flow pattern. The second is the upstream effect of the stator acting on the flow distribution inside the impeller channels. Moreover, in this study, the upstream effect of the volute tongue is significant from vorticity distribution inside the different blade channels. From Fig. 12, it is found that vorticity distribution in blade Channel 2 facing the volute tongue is different from that at other channels as shown in Fig. 10. In Fig. 10, vorticity region $\beta$ shows good coherent on the blade pressure side, however, in Fig. 12a, vorticity region $\beta 1$ detaches from Blade 2, extending into the central zone of the blade channel. In Fig. 12b, with Blade 2 moving away from the volute tongue, vorticity region $\beta 1$ separates completely from Blade 2. Moreover, it experiences a reduction in magnitude due to the mixing effect of the low vorticity magnitude fluid in the central zone of the blade channel, as shown in Fig. $12 \mathrm{~d}$; finally, it would be shed in the wake of the blade trailing edge into the volute casing. With Blade 1 moving close to the volute tongue, the upstream effect of the volute tongue would significantly affect the vorticity distribution inside Blade channel 1. As shown in Fig. 12b, vorticity region $\beta 2$ starts to detach from the pressure side of Blade 1 . With Blade 1 rotating further, vorticity region $\beta 2$ separates and stretches into the central zone of the channel. Finally, it would also undergo a decrease in magnitude and be shed in wake into the volute casing. Therefore, it is concluded that the vorticity distributions in different blade channels show great discrepancy due to the upstream effect of 
the volute tongue acting on the impeller. When the blade channel passes the volute tongue, the vorticity region on the blade pressure side would detach into the central zone of the channel. However, in other blade channels, the vortical structure shows coherent characteristics on the blade pressure sides.

As observed in Fig. 9, it is evident that flow structures at off-design conditions show great discrepancy compared with that at the nominal condition. Fig. 13 presents vorticity distributions at flow rates of $0.2 Q_{d}$ and $1.4 Q_{d}$. At the high flow rate of $1.4 Q_{d}$, the blade inlet angle is much larger than that at the nominal flow rate leading to flow deviating to the blade suction side. Due to the impacting effect of the inflow on the blade suction side, vorticity region $\gamma$ differs significantly from that at a nominal flow rate. At $1.0 Q_{d}$, vorticity region $\gamma$ develops from the blade leading edge almost covering the whole blade channel. However, at $1.4 Q_{d}$, vorticity region $\gamma$ starts to develop at the blade downstream covering half of the blade chord. At the blade trailing edge, vortical structure sheds in the wake, which is in coincidence with that at a nominal flow rate. At a low flow rate of $0.2 Q_{d}$, in contrast with that at $1.4 Q_{d}$, the blade inlet angle is much smaller than that at a nominal flow rate. Flow at the blade leading edge would deviate to the blade pressure side. As observed, high vorticity region $\varepsilon$ develops at the blade leading edge due to the deviating effect of the inflow. Vorticity sheets, at blade pressure and suction sides, are not significant compared with that at the rated condition. As presented in Fig. 9, many small-scale vortices are generated in the central zone of the blade channel, resulting in vorticity magnitude increasing rapidly.

Furthermore, it is found that vortical structures show a significant difference for flow rates 0.2 $Q_{d}$ and $1.4 Q_{d}$ at the near tongue region. At $1.4 Q_{d}$, vortical structure shedding from the blade outlet is eventually cut by the volute tongue and interacts with the tongue intensely. One portion of the vortical structure continues to move to the diffuser exit. However, at a low flow rate of $0.2 Q_{d}$, the cut effect of the volute tongue is not significant, and the whole wake approaching the volute tongue moves to the narrow side of the tongue. The vortical structure at the near tongue region is closely associated with the corresponding absolute velocity distribution as presented in Fig. 14. At a low flow rate of $0.2 Q_{d}$, a significant portion of the fluid leaks from the wide side of the tongue to the narrow side of the volute tongue. In contrast, at a high flow rate of $1.4 Q_{d}$, all fluid tends to move into the diffuser section of the volute casing. Thus, the vortical structure at the near tongue zone is associated with absolute velocity distribution and is significantly affected by the leakage flow direction at the volute tongue region.

\section{CONCLUSIONS}

Rotor-stator interaction and unsteady flow in a low specific speed centrifugal pump are investigated in this study, and some conclusions are carried out.

Angular distributions of pressure amplitudes at $f_{B P F}$ show a typical modulated pattern due to the intense rotor-stator interaction. At nominal flow rate, pressure amplitudes at $f_{B P F}$ along the volute casing show a decreasing tendency in the distant region of the volute tongue, and it is attributed to the increasing gap between the impeller and volute.

Four different patterns of vorticity regions are captured on the mid-span of the impeller. It is evident that the vortical structure, shedding in the wake from the blade exit, interacts intensely with the volute tongue. From a combination analysis of pressure pulsation and vortical structure, it is found that the pressure pulsation amplitude is determined by the corresponding vorticity magnitude. When the blade passes the volute tongue, it is also evident that the upstream effect of the volute tongue has a significant influence on vorticity distribution on the blade pressure side. At off-design flow rates, vortical structures on the blade suction side at $1.4 Q_{d}$, blade leading edge and around the tongue region at $0.2 Q_{d}$ exhibit great discrepancy from that at a nominal flow rate.

For further investigation, experimental investigations consisting of pressure pulsation and unsteady PIV measurements should be conducted to validate the numerical results and provide a comprehensive view of rotor-stator interaction in centrifugal pumps.

\section{ACKNOWLEDGMENT}

The authors gratefully acknowledge the financial support of National Natural Science Foundation of China (51576090, 51476070, 51206063), a Project Funded by the Priority Academic Program Development of Jiangsu Higher Education Institutions (PAPD), and the Research and Innovation Project for College Graduates of Jiangsu Province (KYLX_1036).

\section{REFERENCES}

[1] Rodriguez, C.G., Mateos-Prieto, B., Egusquiza, E. (2014). Monitoring of rotor-stator interaction in a pump-turbine 
using vibration measured with onboard sensors rotating with shaft. Shock and Vibration, vol. 2014, art. ID. 276796, DOI:10.1155/2014/276796.

[2] Kato, C., Mukai, H., Manabe, A. (2003). Large-eddy simulation of unsteady flow in a mixed-flow pump. International Journal of Rotating Machinery, vol. 9, no. 5, p. 345-351, D0l:10.1155/ S1023621X03000320.

[3] Liu, H.L., Wang, K., Kim, H.B., Tan, M.G., (2013). Experimental investigation of the unsteady flow in a double-blade centrifugal pump impeller. Science China Technological Sciences, vol. 56, no. 4, p.812-817, D0l:10.1007/s11431-013-5154-0.

[4] Byskov, R.K., Pedersen, N. (2003). Flow in a centrifugal pump impeller at design and off-design conditions-part II: large eddy simulations. ASME Journal of Fluids Engineering, vol. 125, no. 1, p. 73-83, DOI:10.1115/1.1524586.

[5] Pei, J., Yuan, S.Q., Yuan, J.P. (2013). Fluid-structure coupling effects on periodically transient flow of a single-blade sewage centrifugal pump. Journal of Mechanical Science and Technology, vol. 27, no. 7, p.2015-2023, Dol:10.1007/ s12206-013-0512-1.

[6] Posa, A., Lippolis, A., Verzicco, R., Balaras, E. (2011). Largeeddy simulations in mixed-flow pumps using an immersedboundary method. Computers \& Fluids, vol. 47 , no. 1,p. 33-43, D0l:10.1016/j.compfluid.2011.02.004.

[7] Posa, A., Lippolis, A., Balaras, E. (2015). Large-eddy simulation of a mixed-flow pump at off-design conditions. ASME Journal of Fluids Engineering, vol. 137, no. 10, p. 101302, DOI:10.1115/1.4030489.

[8] Parrondo, J.L., González, J., Fernández-Francos, J. (2002). The effect of the operating point on the pressure fluctuations at the blade passage frequency in the volute of a centrifugal pump. ASME Journal of Fluids Engineering, vol. 124, no. 3, p. 784-790, DOl:10.1115/1.1493814.

[9] Keller, J., Blanco, E., Barrio, R., Parrondo, J. (2014). PIV measurements of the unsteady flow structures in a volute centrifugal pump at a high flow rate. Experiments in Fluids, vol. 55, no. 10, art no.1820, D0l:10.1007/s00348-014-18207.

[10] Wu, Y.L., Yuan, H.J., Shao, J., Liu, S.H. (2009). Experimental study on internal flow of a mini centrifugal pump by PIV measurement. International Journal of Fluid Machinery and Systems, vol. 2, no. 2, p. 121-126, Dol:10.5293/ IJFMS.2009.2.2.121.

[11] Wu, Y.L., Liu, S.H., Yuan, H.J., Shao, J. (2011). PIV measurement on internal instantaneous flows of a centrifugal pump. Science China Technological Sciences, vol. 54, no. 2, p. 270-276, DOl:10.1007/s11431-010-4262-3.

[12] Feng, J.J., Benra, F.K., Dohmen, H.J. (2011). Investigation of periodically unsteady flow in a radial pump by CFD simulations and LDV measurements. ASME Journal of Turbomachinery, vol. 133, no. 1, art. id. 011004, D0l:10.1115/1.4000486.

[13] Yang, S.S., Liu, H.L., Kong, F.Y., Xia, B., Tan, L.W. (2014). Effects of the radial gap between impeller tips and volute tongue influencing the performance and pressure pulsations of pump as turbine. ASME Journal of Fluids Engineering, vol. 136, no. 5, art. no. 054501, Dol:10.1115/1.4026544.

[14] Zhang, N., Yang, M.G., Gao, B., Li, Z., Ni, D. (2015). Experimental investigation on unsteady pressure pulsation in a centrifugal pump with special slope volute. ASME Journal of Fluids Engineering, vol. 137, no. 6, art. no. 061103, Dol:10.1115/1.4029574.

[15] Yang, Z.J., Wang, F.J., Zhou, P.J. (2012). Evaluation of subgridscale models in large-eddy simulations of turbulent flow in a centrifugal pump impeller. Chinese Journal of Mechanical Engineering, vol. 25, no. 5, p. 911-918, D0l:10.3901/ CJME.2012.05.911.

[16] Yamanishi, N., Fukao, S., Qiao, X., Kato, C., Tsujimoto,Y. (2007). LES simulation of backflow vortex structure at the inlet of an inducer. ASME Journal of Fluids Engineering, vol. 129, no .5, p. 587-594, D0l:10.1115/1.2717613.

[17] Bing, H., Cao, S.L. (2014). Parametrization of blade leading and trailing edge positions and its influence on mixed-flow pump performance. Journal of Mechanical Engineering Science, vol. 228, no. 4, p.703-714, DOI:10.1177/0954406213490104.

[18] Gao, Z.X., Zhu, W.R., Lu, L., Deng, J., Zhang, J.G., Wuang, F.J. (2014). Numerical and experimental study of unsteady flow in a large centrifugal pump with stay vanes. ASME Journal of Fluids Engineering, vol. 136, no. 7, art. no. 071101, DOI:10.1115/1.4026477.

[19] Pei, J., Benra, F.K., Dohmen, H.J. (2012). Application of different strategies of partitioned fluid-structure interaction simulation for a single-blade pump impeller. Proceedings of the Institution of Mechanical Engineers. Part E: Journal of Process Mechanical Engineering, vol. 226, no. 4, p. 297-308, Dol:10.1177/0954408911432974.

[20] Barrio, R., Fernández, J., Blanco, E., Parrondo, J. (2011). Estimation of radial load in centrifugal pumps using computational fluid dynamics. European Journal of Mechanics - B/Fluids, vol. 30, no. 3, p. 316-324, D0l:10.1016/j. euromechflu.2011.01.002.

[21] Barrio, R., Blanco, E., Parrondo, J., González, J., Fernández, J. (2008). The effect of impeller cutback on the fluid-dynamic pulsations and load at the blade-passing frequency in a centrifugal pump. ASME Journal of Fluids Engineering, vol. 130, no. 11, art. no. 111102, D0l:10.1115/1.2969273.

[22] Barrio, R., Parrondo, J., Blanco, E. (2010). Numerical analysis of the unsteady flow in the near-tongue region in a volute-type centrifugal pump for different operating points. Computers \& Fluids, vol. 39, no. 5, p. 859-870, D0l:10.1016/j. compfluid.2010.01.001.

[23] Fu, Y., Yuan, J., Yuan, S., Pace, G., d'Agostino, L., Huang, P., Li, X. (2015). Numerical and experimental analysis of flow phenomena in a centrifugal pump operating under low flow rates. ASME Journal of Fluids Engineering, vol. 137, no. 1, art. no. 011102, Dol:10.1115/1.4027142. 